\title{
Problemfall: Entfernung eines gebrochenen kanülierten Tibiamarknagels
}

\author{
A Problematic Case: Removal of a Broken Cannulated Tibial Nail
}

Autoren

Institut
R. Langenhan, N. Reimers, W. Henke, A. Probst

Klinik für Orthopädie, Unfall- und Handchirurgie, Hegau-Bodensee-Klinikum Singen, Gesundheitsverbund Landkreis Konstanz gGmbH

Schlüsselwörter
gebrochener Tibiamarknagel
Ausschlaginstrumentarium
Pseudarthrose
Key words
broken tibial nail
removal device
pseudarthrosis

Bibliografie

DOI http://dx.doi.org/

$10.1055 / \mathrm{s}-0035-1545707$

Online-publiziert 30.04.2015

Z Orthop Unfall 2015; 153:

296-298 ๑ Georg Thieme

Verlag KG Stuttgart · New York .

ISSN 1864-6697

\section{Korrespondenzadresse} Dr. Ronny Langenhan

Klinik für Orthopädie, Unfallund Handchirurgie

HBK Singen

Virchowstraße 10

78224 Singen

Tel.: 07731/890

Fax: 07731/892405

ronnyla@gmx.de

\section{Zusammenfassung}

$\nabla$

Wir stellen eine einfache und sichere Technik der Entfernung eines gebrochenen kanülierten Tibiamarknagels nach ausgebliebener knöcherner Konsolidation einer Unterschenkelschaftfraktur vor. Ein 3-mm-Bohrdraht mit Olive wurde durch das kanülierte proximale und distale Nagelsegment vorgeschoben. Ein 2. an der Spitze angeschliffener 2,5-mm-Draht wurde ebenfalls im distalen Nagelsegment platziert. Der 3-mm-Bohrdraht mit Olive wurde dadurch im distalen Nagelsegment verklemmt und die Olive aus der Achse der Kanülierung des distalen Fragments verdrängt. Beim Herausschlagen des distalen Segments wirkte die Olive wie ein Haken. Durch kräftige retrograde Schläge auf eine den Olivenbohrdorn fixierende Ausschlagzange konnte der gesamte Tibiamarknagel entfernt werden. Durch erneutes Aufbohren der Markhöhle und Reosteosynthese des Tibiaschafts mittels eines AO-Standardnagels heilte die Fraktur innerhalb von 12 Monaten aus.

\section{Einleitung}

$\nabla$

Die intramedulläre Stabilisation von dislozierten Tibiaschaftfrakturen ist der Goldstandard und führt regelhaft zur Ausheilung der Verletzung. Trotzdem kann es in einigen Fällen zu einer verzögerten Heilung oder Pseudarthrose kommen, was eine Überlastung des Implantats bedeutet. Dies kann zu einem Nagelbruch führen [1]. Verschiedene Techniken zur Entfernung des distalen Segments eines gebrochen Marknagels wurden bereits beschrieben. Einige sind sehr zeitaufwendig, andere bedeuten große operative $\mathrm{Zu}-$ gänge oder benötigen spezielle Instrumente wie lange Haken, Führungsdrähte, Cerclagen, lange Zangen oder Bohrer [2-9]. Idealerweise werden Extraktionshaken verwendet, die durch das kanülierte Nagelfragment eingeschoben und retrograd

\section{Abstract \\ $\nabla$}

We present a case illustrating a simple and safe technique for the removal of a broken cannulated tibial nail after a pseudarthrosis of a lower leg shaft fracture. A $3 \mathrm{~mm}$ Ball-Tip guide wire was inserted into the proximal and the distal segment of the nail. A $2.5 \mathrm{~mm}$ tip-flattened second wire was forwarded into the distal segment pushing the Ball-Tip guide wire out of the axis and blocking it. This way the Ball-Tip could act as a hook and consecutively could be knocked back with an impactor forceps removing the complete nail. An exchange nailing was performed with a reamed AO standard nail and the further course was uneventful with a healed fracture after 12 months. mithilfe des Hakens aus der Markhöhle geborgen werden. Diese Technik kann jedoch fehlschlagen, wenn der Haken sich nicht am Fragmentende des Nagels verankern lässt. Wir stellen eine weitere einfache und sichere Technik zur Entfernung eines kanülierten gebrochenen Tibiamarknagels vor.

\section{Kasuistik}

Eine 23-jährige Patientin stürzte mit dem Motorrad und zog sich eine zweitgradig offene, dislozierte mehrfragmentäre Fraktur des Tibia- und Fibulaschafts im mittleren Drittel (AO-Typ 42C1.1) zu. Nach primärer Stabilisation der Fraktur durch einen Fixateur externe (ProCallus ${ }^{\circledR}$, Firma Orthofix GmbH, Germany) wurde die Fraktur 12 Tage später intramedullär mit einem aufge- 


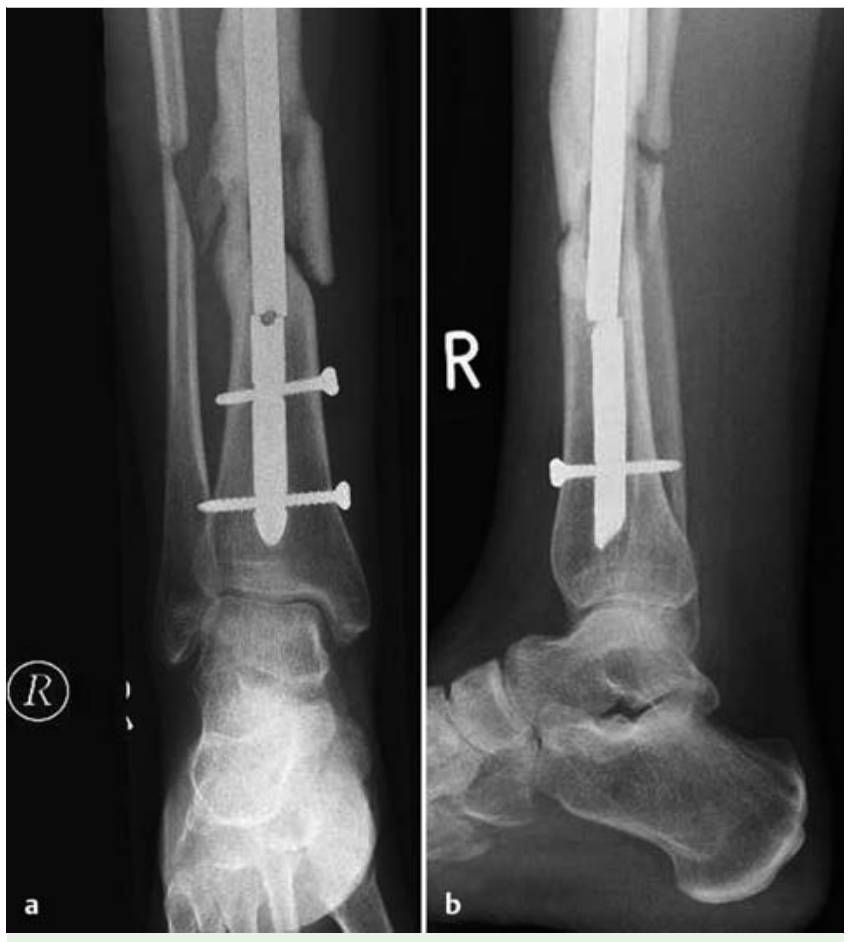

Abb. 1 a und $\mathbf{b}$ Röntgenbild des rechten distalen Unterschenkels a.-p. (a) und lateral (b) mit atropher Pseudarthrose der Unterschenkelschaftfraktur und gebrochenem Tibiamarknagel.

bohrten Tibiamarknagel (Sirus ${ }^{\circledR}$ Tibiamarknagel, Firma Zimmer, Durchmesser 9,3 mm, Länge $360 \mathrm{~mm}$ ) definitiv versorgt. Zehn Monate später hatte sich eine atrophe Pseudarthrose mit einem Nagelbruch $3,5 \mathrm{~cm}$ unterhalb der Pseudarthrose ausgebildet (๑ Abb. 1).

Bei der operativen Revision wurde nach der Entfernung der proximalen Verschlusskappe und der Verriegelungsbolzen ein 3-mm-Bohrdorn mit Olive durch das proximale und das distale Fragment des Nagels vorgeschoben. Die Olivenspitze wurde außerhalb des distalen Nagelfragments platziert. Zusätzlich wurde ein 2. an der Spitze angeschliffener 2,5-mm-Draht (Firma Marquardt Medizintechnik, Spaichingen/Deutschland) über das proximale in das distale Nagelsegment eingeführt und mit Hammerschlägen im distalen Nagelsegment verklemmt. Der meißelförmige Anschliff des Drahtes bewirkt zum einen die Verklemmung der beiden Drähte in der Kanülierung des distalen Segments, zum anderen verdrängt er die Olivenspitze aus der Achse der Kanülierung des distalen Segments, sodass die Olive beim Zurückschlagen der Drähte wie ein Haken fungiert ( Abb. 2). Mittels Bündelnagelzange (nach Hackethal ${ }^{\circledR}$, Ulrich Medical, Germany) wurde der so im distalen Nagelsegment verklemmte Olivenbohrdraht nach proximal geschlagen, wodurch beide Nagelsegmente entfernt werden konnten ( $\bullet$ Abb. 2). Der Tibiamarkraum wurde erneut aufgebohrt und mit einem AO Standard Nage ${ }^{\circledR}$ (Firma Synthes, Durchmesser $11 \mathrm{~mm}$, Länge $360 \mathrm{~mm}$ ) stabilisiert. 12 Monate postoperativ war die Pseudarthrose ausgeheilt.

\section{Diskussion \\ $\nabla$}

Die Versorgung von Unterschenkelschaftfrakturen mit der Tibiamarknagelosteosynthese führt regelhaft zur Ausheilung. Bei verzögerter ossärer Konsolidation birgt die erhöhte Belastung des Marknagels im Bereich der Frakturzone das Risiko eines Implantatversagens [1]. Die Inzidenz eines Marknagelbruches wird mit 1-3\% angegeben [3]. Bei ausgebliebener ossärer Konsolidation und vorliegendem Marknagelbruch besteht die Notwendigkeit der Reosteosynthese. Verschiedene Techniken der Entfernung des distalen Nagelsegments wurden bereits beschrieben. Einige sind zeitintensiv, andere benötigen zusätzliche Zugangswege und/oder Spezialinstrumente wie lange Haken, Führungsdrähte, Cerclagen, lange Zangen oder Bohrer [2-9].

Levine und Georgiadis fädelten bei einem gebrochenen kanülierten Tibiamarknagel das distale Nagelsegment mit einem flexiblen Olivendraht von retrograd über einen seperaten Knochenkanal vom Malleolus medialis auf und konnten so das gebrochene Nagelsegment nach proximal über den Standardzugang herausschlagen [9]. Neben dem zusätzlichen Weichteiltrauma und der Schwächung des medialen Malleolus ist diese Technik nur möglich, wenn das Nagelende ausreichend weit von der distalen Tibiagelenkfläche platziert wurde.

Die von Schmidgen et al. beschriebene Technik stellt eine weitere Möglichkeit der Entfernung sowohl eines soliden als auch eines kanülierten abgebrochenen distalen Nagelsegments dar [8]. Hier wurde nach Entfernung des proximalen Nagelendes die Markhöhle bis zum distalen Nagelanteil auf $11 \mathrm{~mm}$ aufgebohrt. Durch Manipulation über ein distales knöchernes Verriegelungsloch mittels kleinem gebogenem Raspatorium konnte das Nagelende in eine Position gebracht werden, in welcher ein von proximal eingeführter, gebogener und zurechtgefeilter AO-Ausschlaghaken in ein Verriegelungsloch des distalen Nagelsegments eingehakt und dieses so nach proximal extrahiert werden konnte. Vo-
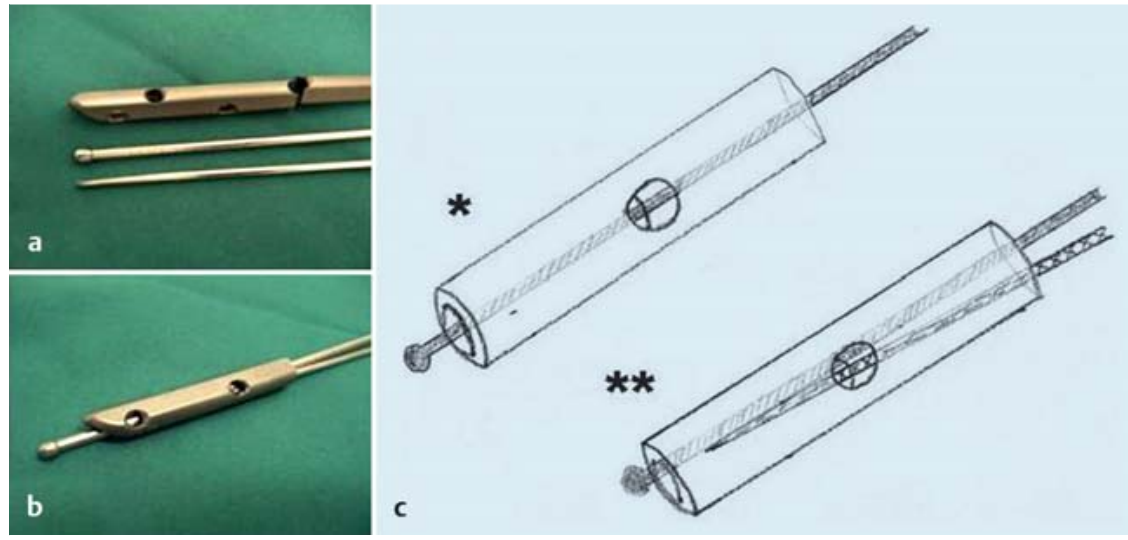

Abb. 2 a bis c Fotografie des entfernten, gebrochenen distalen Nagelendes, des 3-mm-Bohrdrahtes mit Olive und des an der Spitze meißelförmig angeschliffenen 2,5-mm-Drahtes (a). Darstellung des Verblockungsmanövers mit dem entfernten Nagelsegment als Fotografie (b) und als Schemazeichnung (c). Einführen des Olivenbohrdrahtes durch das distale Nagelsegment $\left({ }^{*}\right)$. Im 2. Schritt wird der an der Spitze meißelförmig angeschliffene Draht ebenfalls eingeschlagen, verklemmt so den Olivenbohrdraht langstreckig im distalen Nagelsegment und drängt die Olive aus der Achse der Kanülierung, sodass diese Olive als Haken fungiert $\left({ }^{* *}\right)$. 
raussetzung für diese Methode bleibt das Aufbohren der proximalen Markhöhle und die Mobilisation des distalen Nagelsegments, damit eine Kippung in der Markhöhle möglich ist und der Ausschlaghaken zwischen Kortikalis und Nagel Platz findet. Middleton et al. beschreiben eine vergleichbare Technik zu der unseren bei der Entfernung eines gebrochenen kanülierten Femurmarknagels [10]. Hier wurde der initial eingebrachte Olivenbohrdraht mit 2 weiteren Olivenbohrdrähten im gebrochenen distalen Nagelsegment verblockt. Durch kräftige retrograde Schläge auf eine den primären Olivenbohrdraht fixierende Ausschlagzange konnte das Nagelsegment entfernt werden. Zuvor wurde der Markraum bis zum distalen Nagelsegment durch Aufbohren erweitert. Da viele kanülierte Tibiamarknägel deutlich kleinere Innendurchmesser haben als Femurmarknägel ist diese Technik nicht zwingend auf die Entfernung eines kurzen gebrochenen distalen Tibiamarknagelsegments übertragbar. Durch die von uns beschriebene Technik können hingegen auch sehr dünne kanülierte Nägel erfolgreich entfernt werden.

\section{Schlussfolgerung}

$\nabla$

Die beschriebene Methode zur Entfernung eines gebrochenen kanülierten Tibiamarknagels ist technisch einfach durchzuführen, benötigt keine weiteren Zugänge und bis auf einen an der Spitze angeschliffenen Draht keine weiteren Spezialinstrumente. Durch unterschiedliche Drahtdurchmesser können auch dünne kanülierte Nagelsegmente entfernt werden.

Interessenkonflikt: Nein

\section{Literatur}

1 Zimmerman KW, Klasen HJ. Mechanical failure of intramedullary nails after fracture union. J Bone Joint Surg Br 1983; 65: 274-275

2 Incavo SJ, Kristiansen TK. Retrieval of a broken intramedullary nail. Clin Orthop Relat Res 1986; 210: 201-202

3 Franklin JL, Winquist RA, Benirschke SK et al. Broken intramedullary nails. J Bone Joint Surg Am 1988; 70: 1463-1471

4 Gregory PR jr. Removal of a broken solid-core intramedullary femoral nail using both antegrade and retrograde starting points. Orthopedics 1997; 20: 1087-1089

5 Khan M, Schranz PJ, Ward MW. Removal of a broken intramedullary tibial nail using a hand reamer. Injury 1997; 28: 693-694

6 Charnley GJ, Farrington WJ. Laparoscopic forceps removal of a broken tibial intramedullary nail. Injury 1998; 29: 489-490

7 Marwan M, Ibrahim M. Simple method for retrieval of distal segment of the broken interlocking intramedullary nail. Injury 1999; 30: 333-335

8 Schmidgen A, Naumann 0 , Wentzensen A. [A simple and rapid method for removal of broken unreamed tibial nails]. Unfallchirurg 1999; 102: 975-978

9 Levine JW, Georgiadis GM. Removal of a broken cannulated tibial nail: a simple intramedullary technique. J Orthop Trauma 2004; 18: 247-249

10 Middleton RG, McNab IS, Hashemi-Nejad A et al. Multiple guide wire technique for removal of the short distal fragment of a fractured intramedullary nail. Injury 1995; 26: 531-532 